\title{
Interparticle magnetic correlations in dense Co nanoparticle assemblies
}

\author{
Jeffrey B. Kortright, ${ }^{1}$ Olav Hellwig, ${ }^{2}$ Shouheng Sun, ${ }^{3}$ and Eric E. Fullerton, ${ }^{2}$ \\ ${ }^{1}$ Lawrence Berkeley National Laboratory, Berkeley, California 94720 \\ 2 San Jose Research Center, Hitachi Global Storage Technologies, \\ 650 Harry Road, San Jose, California 95120 \\ ${ }^{3}$ IBM T. J. Watson Research Center, Yorktown Heights, New York 10598
}

Resonant magnetic x-ray scattering from dense self-assemblies of 9-nm diameter $\varepsilon$-Co and $h c p$-Co particles is reported. For lower anisotropy $\varepsilon$-Co we find remanent magnetic scattering that is significantly enhanced, indicating preferred inter-particle moment orientations of both antiferromagnetic and ferromagnetic character. This interactionmediated collective behavior is consistent with dipolar fields and exists well above the isolated particles blocking temperature where thermal activation is operative, suggesting that magnetostatic super-spin waves exist in such systems.

PACS: $75.25 .+\mathrm{z}, 75.50 . \mathrm{Tt}, 75.75 .+\mathrm{a}, 75.20 .-\mathrm{g}$ 
Magnetic nanoparticles with a protective organic shell self-assemble into densely packed arrays $[1,2]$. Close interparticle spacing in dense arrays increases the dipolar interaction energy to compete with single-particle anisotropy, Zeeman, and thermal energies $[3,4]$. Macroscopic magnetic measurements $[5,6,7,8,9,10]$ and micromagnetic modeling studies $[3,4,11,12]$ suggest the importance of dipolar interactions, that are thought to lead to collective behavior below the blocking temperature of otherwise superparamagnetic particles $[3,11,6]$. Few measurements, however, are sensitive to interactions at interparticle length scales where dipolar fields should be most pronounced.

The magnetostatic energy between dipoles $\boldsymbol{\mu}_{i}$ and $\boldsymbol{\mu}_{j}$ separated by $\mathbf{r}_{i j}$, $E_{i, j}^{D}=\left(\boldsymbol{\mu}_{i} \cdot \boldsymbol{\mu}_{j}\right) / r_{i j}^{3}-3\left(\boldsymbol{\mu}_{i} \cdot \mathbf{r}_{i j}\right)\left(\boldsymbol{\mu}_{j} \cdot \mathbf{r}_{i j}\right) / r_{i j}^{5}$, has minima ranging from ferromagnetic (F) alignment when $\boldsymbol{\mu}_{i}, \boldsymbol{\mu}_{j}$, and $\mathbf{r}_{i j}$ are collinear, to antiferromagnetic (AF) alignment when collinear $\boldsymbol{\mu}_{i}$ and $\boldsymbol{\mu}_{j}$ are orthogonal to $\mathbf{r}_{i j}$. Orientations taken by interacting dipoles in dense 3-D samples are thus not simple, often involving frustration and spin-glass behavior. The correlation function $\xi=\left\langle\boldsymbol{\mu}_{i} \cdot \boldsymbol{\mu}_{j}\right\rangle$ is a useful descriptor for the ensemble of local dipole-dipole correlations, since it has positive and negative extrema for these same collinear $\mathrm{F}$ and transverse AF order, respectively [3].

In this letter we study dense, self-assembled systems of $9 \mathrm{~nm}$ superparamagnetic Co particles using resonant magnetic x-ray scattering at wavelengths short enough to resolve nearest neighbor structure and provide sensitivity to $\xi$. Combining applied fields and variable incident polarization we confirm theoretical predictions of charge-charge, magnetic-magnetic, and charge-magnetic contributions to this scattering. Pure magnetic 
intensity is isolated via field differences and compared with models indicating that dipolar interactions can favor specific, not random, $\boldsymbol{\mu}_{i} \cdot \boldsymbol{\mu}_{j}$ configurations in low anisotropy particles. We observe moments oriented out of the sample plane that have statistacally significant tendencies toward AF order dispersed in the plane and $\mathrm{F}$ order normal to the plane, even at $298 \mathrm{~K}$, as well as strong sensitivity to particle anisotropy. This dynamic order suggests the presence of magnetostatic super-spin waves.

Densely packed samples are formed from hexane dispersions of $9 \mathrm{~nm}$ diameter $( \pm$ 5\%) Co particles of lower anisotropy $\varepsilon$ and higher anisotropy $h c p$ phases $[13,14]$. Isolated particles of each phase are superparamagnetic (SPM) at $298 \mathrm{~K}$. Concentrated single-phase samples were prepared by allowing a drop of suspended particles to dry on $150 \mathrm{~nm}$ thick $\mathrm{Si}_{3} \mathrm{~N}_{4}$ membranes for transmission scattering and absorption measurements. Scattered intensity $I(q, H)$ was measured in symmetric transmission geometry (Fig. 1a inset) to position the scattering vector $\mathbf{q}$ in the membrane plane [15], using both linear $\left(I_{\text {lin }}\right)$ and circular $\left(I_{ \pm}\right)$polarization with variable applied field $\mathbf{H}$ perpendicular to the membrane at both 298 and $32 \mathrm{~K}$. It is convenient to define a coordinate system with $\mathbf{x}$ along the sample normal (and $\mathbf{H}$ ) and $\mathbf{z}$ along $\mathbf{q}$. Several samples of each phase were observed to have similar behavior; here we present detailed results from one sample of each phase.

Transmission absorption spectra across the Co $L_{2,3}$ edges provide valuable, albeit spatially averaged, information. Measurements at $298 \mathrm{~K}$ using circular polarization and near saturating field, $H_{\text {sat }}= \pm 0.5 \mathrm{~T}$, yield the imaginary parts, $f_{2}$, of the resonant magnetic $\left(f_{m}\right)$ and charge $\left(f_{c}\right)$ atomic scattering factors $f_{m / c}=f_{m / c, 1}+i f_{m / c, 2}[16,17]$, seen for $\varepsilon$-Co in Fig. 2. The real parts, $f_{1}$, are obtained via Kramers-Kronig transformation. The 
absorption step in $f_{c, 2}$ indicates that aggregated regions are $\sim 20$ particles thick. The strength of the $f_{c, 2}$ lines are comparable to those in bulk Co [18]. The size of $f_{m, 2}$ relative to $f_{c, 2}$ lines is roughly 2-3 smaller than for bulk $\mathrm{Co}$, so the average moment per atom in the particles is correspondingly smaller $\left(\sim 0.5-0.8 \mu_{B}\right.$ in the particle vs. $1.7 \mu_{B}$ in bulk Co). A $9 \mathrm{~nm}$ particle contains $\sim 34,000$ atoms, and so has moment $|\boldsymbol{\mu}| \sim 22,000 \mu_{B}$. The particle scattering factors are thus 34,000 times $f_{c}$ and $f_{m}$, although below we rescale and take atomic values to represent particles. Intensities of pure magnetic and charge scattering are in the inset; $\left|f_{m}\right|^{2} /\left|f_{c}\right|^{2}=0.026$ for $\varepsilon$-Co at the $L_{3}$ line, where all data here were collected. Similar spectra for the $h c p$-Co sample yield slightly larger $f_{c}$ and $f_{m}$ values at the $L_{3}$ peak, and intensity ratio 0.037 .

Interparticle chemical and magnetic structure is obtained from $I_{\text {lin }}(q)$ for $\varepsilon$ - and $h c p$-Co samples (Fig. 1a \& 1b). Each sample exhibits a diffuse ring peaking at $q_{p e a k}=$ $0.54 \mathrm{~nm}^{-1}$ arising from interparticle interference. These data have been normalized for slit size effects and by the spherical particle form factor to facilitate quantitative comparison of intensity features vs. $q$. The characteristic length $2 \pi / q_{\text {peak }}=11.6 \mathrm{~nm}$ confirms dense assemblies, and that the organic ligand shells remain intact to preclude interparticle exchange interactions. The peak widths $\Delta q=0.096 \mathrm{~nm}^{-1}$ and $0.12 \mathrm{~nm}^{-1}$ for the $\varepsilon$ - and $h c p$-Co samples, respectively, are consistent with relatively disordered particle assemblies. Both samples exhibit increasing intensity as $q \rightarrow 0$ indicating that the assemblies are not uniform at length scales $>500 \mathrm{~nm}$.

Magnetic structure is obtained from the difference between the two $q$ scans measured at $H=0$ and $H_{\text {sat }}$. For each sample, $I_{\text {lin }}\left(H_{\text {sat }}\right)$ is systematically larger than $I_{l i n}(H$ $=0)$ at $q_{\text {peak }}$, and smaller at lower $q$. The difference $\Delta I_{\text {lin }} \equiv I_{\text {lin }}(H=0)-I_{\text {lin }}\left(H_{\text {sat }}\right)$, in 
Figs. $1 \mathrm{c} \& 1 \mathrm{~d}$, is clearly magnetic in origin and easily understood qualitatively. At $H_{\text {sat }}$ all moments are oriented along $\mathbf{H}(\| \mathbf{x})$, yielding commensurate charge and magnetic scattering amplitudes for which magnetic intensity adds to the charge intensity at $q_{\text {peak }}$. At $H=0$ and $298 \mathrm{~K}$ the sample has no net moment (see below), and the commensurate magnetic intensity at $q_{\text {peak }}$ is reduced. The increase in remanent intensity below $q_{\text {peak }}$ arises from magnetic correlations that are longer than the interparticle spacing. This scattering is analogous in origin to magnetic scattering at the $1 / 2$ order position of an AFcoupled magnetic superlattice [19]. We thus refer to these negative and positive trends in $\Delta I_{\text {lin }}$ as the $\mathrm{F}$ and AF peaks. Their areas at $298 \mathrm{~K}$ are in Table 1, along with the same quantities for the $\varepsilon$-Co sample at $32 \mathrm{~K}$, normalized by the $1^{\text {st }}$ order peak in $I_{\text {lin }}(H=0)$ that approximates the pure charge interference intensity. For the $h c p$-Co sample the AF intensity increase is substantially smaller than the $\mathrm{F}$ decrease. For $\varepsilon$-Co the AF increase is $\sim 2$ times larger than the $\mathrm{F}$ decrease. These large differences in the relative strengths of the $\mathrm{F}$ and $\mathrm{AF}$ peaks indicate very different remanent magnetic structures between samples, and we argue that dipolar interactions yield significant moment correlations in the latter case.

To understand more quantitatively if and how dipolar interactions influence $\Delta I_{\text {lin }}$, we develop a simple scattering model. Adopting the circular polarization basis, the scattering factor simplifies to $f_{ \pm}=p_{c} f_{c} \pm p_{m} f_{m}$, where \pm refers to opposite helicity and $p_{c}=\left(\mathbf{e}_{f}^{*} \cdot \mathbf{e}_{o}\right)$ and $p_{m}=i\left(\mathbf{e}_{f}^{*} \times \mathbf{e}_{o}\right) \cdot \mathbf{m}$ are the charge and magnetic polarization prefactors depending on scattering angle $2 \theta$, incident polarization $\mathbf{e}_{o}$, and magnetization direction $\mathbf{m} \equiv \boldsymbol{\mu} /|\boldsymbol{\mu}|[16,15]$. Limiting cases for $p_{m}$ correspond to longitudinal $(\mathbf{m} \| \mathbf{x})$, transverse $(\mathbf{m} \| \mathbf{y})$, and polar $(\mathbf{m} \| \mathbf{z})$ moments, for which $p_{m} \cong \pm 0.999, \pm 0.07 i$, and 0 , 
respectively, at $q_{\text {peak. }}$ In the present geometry we are most sensitive to longitudinal $\mathbf{m}$. The AF peaks in $\Delta I_{\text {lin }}$ thus imply AF alignment of longitudinal $\mathbf{m}$ separated along $\mathbf{z}$, for which $\xi<0$ consistent with $E^{D}$. Even uncorrelated, randomly oriented SPM particles would have some statistical probability of exhibiting AF (or F) alignment along $\mathbf{x}$. To determine if dipolar interactions yield correlated moments, we thus must determine whether measured AF peaks are stronger than predicted for randomly oriented $\mathbf{m}_{i}$.

In the Born approximation the ensemble has scattering amplitude $a_{ \pm}=\sum_{i} f_{i \pm} \exp \left[i \mathbf{q} \cdot \mathbf{r}_{i}\right]$ and intensity

$$
\begin{aligned}
& I_{ \pm}(q, \omega, H)=\sum_{i} \sum_{j} f_{i \pm}^{*} f_{j \pm} \exp \left[i \mathbf{q} \cdot \mathbf{r}_{i j}\right] \\
& =f_{c}^{2} s_{c-c}+f_{m}^{2} s_{m-m} \pm 2\left(f_{2 c} f_{1 m}-f_{1 c} f_{2 m}\right) s_{c-m}
\end{aligned},
$$

where sums are over all particles, + and - refer to right and left circular polarization, and partial structure factors $s_{c-c}(q), s_{m-m}(q, \omega, \mathrm{H})$, and $s_{c-m}(q, \omega, \mathrm{H})$ describe the spatial distribution of charge-charge, magnetic-magnetic, and charge-magnetic correlations, respectively. The $q$-dependent spherical particle form factors are not explicitly included, as the data have already been normalized by them. $p_{c}$ and $p_{m}$ are included in the $s_{i-j}$, so that any temporal fluctuations of $\mathbf{m}_{i}$ yield frequency $(\omega)$ dependence only in $s_{m-m}$ and $s_{c-}$ $m$. Since $s_{c-m}$ and $s_{m-m}$ are odd and even in helicity, it follows that $I_{+}-I_{-}=4\left(f_{2 c} f_{1 m}-f_{1 c} f_{2 m}\right) s_{c-m}$ gives only the cross-term, as noted in Ref. [20]. It also follows that for linear polarization $I_{l i n} \equiv\left(I_{+}+I_{-}\right) / 2=f_{c}^{2} s_{c-c}+f_{m}^{2} s_{m-m}$.

Hysteresis loops of $I_{+}, I_{-}$, and $I_{\text {lin }}$ measured at $q / q_{\text {peak }}=1.0$ and 0.32 for the $\varepsilon-\mathrm{Co}$ sample (Fig. 3) confirm this model. The identity $I_{+}+I_{-} \equiv 2 I_{\text {lin }}$ and the anti-symmetry of $I_{+}-I_{-}$hold for all $q$ and for both samples. We see that $I_{\text {lin }}$, rather than $I_{+}-I_{-}$, is 
most appropriate to study dipolar interactions that necessarily involve only pure magnetic correlations. $\Delta I_{l i n}(q)=f_{\mathrm{m}}{ }^{2} \Delta s_{m-m}(q)$ isolates the field dependent changes in $s_{m-m}$ since the charge scattering is independent of $H$. The AF scattering in $\Delta I_{\text {lin }}(q)$ is seen as a pronounced remanent peak in the loops at $q / q_{\text {peak }}=0.32$, while the $\mathrm{F}$ loss peak at $q / q_{\text {peak }}=$ 1.0 is within the size of the data symbols since it sits atop the much larger charge peak. At saturation all $\mathbf{m}_{i} \| \mathbf{x}, \mathbf{m}_{i} \cdot \mathbf{m}_{j} \cong 1$, and $\xi \cong+1$ for all orientations of $\mathbf{r}_{\mathrm{ij}}$. Here, $p_{c} \cong 1 \cong p_{m}, s_{c-c}(q) \cong s_{m-m}(q) \equiv s(q)$, and $I_{l i n}(q) \cong\left(f_{c}^{2}+f_{m}^{2}\right) s(q)$, so that charge and magnetic scattering are indeed commensurate. From above we know that $f_{m}{ }^{2}$ is 2.6 and $3.7 \%$ of $f_{c}^{2}$ at $q_{p e a k}$ for the $\varepsilon$ - and $h c p$-Co samples, respectively. Thus saturation, with known relative strengths of pure charge and magnetic scattering, is the natural reference from which to interpret changes in $s_{m-m}$ with $H$.

At remanence the $\mathbf{m}_{i}$ orientations are unknown and we interpret $\Delta I_{\text {lin }}(q)$ in terms of $\left\langle\mathbf{m}_{i} \cdot \mathbf{m}_{j}\right\rangle$ and $E^{D}$. With limited knowledge of the samples' disordered short- and intermediate-range order, rather than consider explicit 3-D structural models we consider trends in pair-wise $\mathbf{m}_{i} \cdot \mathbf{m}_{j}$ contributions to the magnetic intensity. Consider first the loss of intensity at remanence at $q_{\text {peak }}$. If individual $\mathbf{m}_{i}$ are uncorrelated and equally likely to take all orientations, the pure magnetic intensity at $q_{p e a k}$ is $1 / 6$ that at saturation. For such uncorrelated moments, the probability for two particles exhibiting AF alignment with $\mathbf{m} \| \mathbf{x}$ is identical to that for $\mathrm{F}$ alignment, so the pure AF intensity at $q_{p e a k} / 2$ is also $1 / 6$ of the saturated magnetic intensity at the $1^{\text {st }}$ order peak. Thus, for such uncorrelated $\mathbf{m}_{i}$, the F loss peak would contain $5 / 6$ of the saturated magnetic intensity at the $1^{\text {st }}$ peak, and the AF peak $1 / 6$ of the same amount. Comparing the observed intensities of the $\mathrm{F}$ and AF peaks (Fig. 1c \& 1d, and Table 1), we see that the F peak is 4 times larger than 
the AF peak for the $h c p$ sample; close enough to the uncorrelated prediction for us to conclude that no or only minimal magnetic correlations exist in this sample. For the $\varepsilon$ sample, the AF peak is 2 times larger than the F peak, clearly indicating that remanent $\mathrm{AF}$ correlations are statistically stronger than expected for random moment orientations.

The moments contributing to the AF peak for $\varepsilon$-Co have $\mathbf{m}_{i}, \mathbf{m}_{j} \| \mathbf{x}$ and $\mathbf{r}_{i j} \| \mathbf{z}$. For these moments $\xi<0$ consistent with $E^{D}$, implying that dipolar interactions are indeed responsible for this correlated behavior. The AF intensity increases slowly with decreasing $q$ well below the $1 / 2$ order position before being overshadowed by chemical scattering from sample heterogeneity, indicating that $\mathrm{AF}$ correlations range significantly beyond nearest neighbors.

Several other features about the strong AF correlations are noteworthy. First, they exist at $298 \mathrm{~K}$, well above the blocking temperature $T_{B}$ for isolated $9 \mathrm{~nm}$ particles. Thus, while collective, dipolar-mediated behavior has been discussed before for $T<T_{B}[3,5,8]$, here we have direct evidence for interaction-mediated collective behavior well above $T_{B}$.

Second, $\Delta I_{\text {lin }}$ probes magnetic structure along in-plane $\mathbf{q}$ and averages over the thickness of the sample. The observation of strong AF correlations thus suggests that locally $\int\left(\mathbf{m}_{i} \cdot \mathbf{x}\right) d x \neq 0$, i.e., that some degree of local $\mathrm{F}$ order exists normal to the sample surface. This mixed, oriented AF and F order is again consistent with the pairwise $E^{D}$ expression, and indicative of strong dipolar coupling.

Third, the observations generally support the notion of AF effective fields often assumed in modeling long-range magnetostatic interactions [3,4]. While reassuring, the results here suggest that modeling studies may miss interesting effects (e.g., mixed, 
oriented $\mathrm{AF}$ and $\mathrm{F}$ order) by limiting explicit evaluation of interactions to, typically, 3-4 particle radii.

Fourth, the observation of strong AF correlations with $\mathbf{m} \| \mathbf{x}$ is at odds with dipole-mediated in-plane anisotropy expected for thin film samples. We repeated remanent measurements following in-plane, not perpendicular, saturation, and found no decrease in the AF peak. The remanent AF correlations are thus independent of field history and in this sense characterize the ground state at $T$. While our measurements give no direct knowledge of $\left\langle\mathbf{m}_{i} \cdot \mathbf{m}_{j}\right\rangle$ correlations for which $\mathbf{m}_{i}$ and $\mathbf{m}_{j}$ are in-plane, all correlations we do measure are consistent with magnetostatic energy minimization over intermediate- and long-ranges mediated by short-range dipolar interactions.

Finally, we have yet to discuss thermally activated dynamics (fluctuating or relaxing $\mathbf{m}_{i}$ ) since our time-average measurements have little sensitivity to such motion. While isolated $9 \mathrm{~nm}$ Co particles are SPM at $298 \mathrm{~K}$, it is conceivable that collective dipolar coupling stabilizes moments against thermal fluctuations. Scattering hysteresis loops above and below $T_{B}$ for the $\varepsilon$-Co sample (Fig. 4) address this possibility. These loops were measured using circular polarization $\left(I_{+}\right)$at $q_{\text {peak }}$, and thus predominantly reflect the cross term $s_{c-m}$. The lack of hysteresis at $298 \mathrm{~K}$ is direct evidence that thermal activation obtains at this $T$. The loop shape at $298 \mathrm{~K}$ can be fit with $L(x)=\operatorname{coth}(x)-1 / x$ where $x=\mu H / k T$, yielding $\mu \cong 4000 \mu_{B}$, much smaller than the $22,000 \mu_{B}$ determined from XMCD above. Some hysteresis is observed at $32 \mathrm{~K}$. The lines give $L(x)$ for $\mu=22,000 \mu_{B}$ at both temperatures. These discrepancies between measured and ideal SPM behavior, and the lack of $H / T$ scaling, are further evidence for dipolar interactions of AF character even at $298 \mathrm{~K}$. The coexistence of thermal activation and 
collective magnetism at $298 \mathrm{~K}$ indicates that correlated thermal motion in the form of magnetostatic super-spin waves may at least partially describes the remanent state. Note that the $\Delta I_{l i n} \mathrm{AF}$ peak for the $\varepsilon$ sample increases only slightly at $32 \mathrm{~K}$ (Table 1), indicating that interaction-mediated collective behavior is nearly as strong at $298 \mathrm{~K}$ as at $32 \mathrm{~K}$.

In conclusion, $\Delta I_{\text {lin }}$ isolates changes in pure magnetic scattering involving moments normal to the substrate that is compared to predictions for non-interacting moments taking random orientations with respect to each other. For the lower anisotropy $\varepsilon$-Co sample, stronger than random AF correlations dispersing along in-plane directions coexist with local F correlations normal to the sample implying dipolar mediated collective behavior, even well above the blocking temperature of individual particles. For the higher anisotropy $h c p$-Co sample, results are more consistent with randomly oriented, uncorrelated moments, suggesting that a random distribution of anisotropy axes more strongly influences moment orientations than dipolar interactions in this sample. The coexistence of mixed AF/F correlations with thermal activation argue against a spinglass description for the $\varepsilon$ sample, in favor of dipolar order in which magnetostatic super-spin waves preserve time-average order with no net moment. The high magnetic sensitivity of resonant $\mathrm{x}$-ray scattering at interparticle length scales in small sample volumes, together with the potential to extend these measurements in to the dynamic regime using coherent scattering [21], point to opportunities to better establish the role of competing energies in a variety of nanoscale magnet assemblies.

Work at LBNL and the ALS was supported by the Director, Office of Science, Office of Basic Energy Sciences, under DOE Contract No. DE-AC03-76SF00098. 


\section{References}

1 S. Sun, C. B. Murray, D. Weller, L. Folks, and A. Moser, Science 287, 1989 (2000).

2 V. F. Puntes, K. M. Krishnan, and A. P. Alivisatos, Science 291, 2115 (2001).

3 J. L. Dormann, D. Fiorani, and E. Tronc, Adv. Chem. Phys. 98, 283 (1997).

4 R. W. Chantrell, N. Walmsley, J. Gore, and M. Maylin, Phys Rev. B 63, 024410 (2000).

5 W. Luo, S. R. Nagel, T. F. Rosenbaum, and R. E. Rosensweig, Phys. Rev. Lett. 67, 2721 (1991).

6 T. Jonsson, P. Svedlindh, and M. F. Hansen, Phys. Rev. Lett. 81, 3976 (1998).

7 P. Poddar, J. L. Wilson, H. Srikanth, D. F. Farrell, and S. A. Majetich, Phys. Rev. B 68, 214409 (2003).

8 G. A. Held, G. Grinstein, H. Doyle, S. Sun, and C. B. Murray, Phys. Rev. B 64, 012408 (2001).

9 H. Mamiya, I. Nakatani, and T. Furubayashi, Phys. Rev. Lett. 82, 4332 (1999).

10 Y. Sun, M. B. Salamon, K. Garnier, and R. S. Averback, Phys. Rev. Lett. 91, 167206 (2003).

11 J.-O. Andersson, C. Djurberg, T. Jonsson, P. Svedlindh, and P. Nordblad, Phys. Rev. B. 56, 13983 (1997).

12 J. Garcia-Otero, M. Porto, J. Rivas, and A. Bunde, Phys. Rev. Lett. 84, 167 (1999).

13 S. Sun and C. B. Murray, J. Appl. Phys. 85, 4325 (1999).

14 C. B. Murray, S. Sun, H. Doyle, T. Betley, MRS Bulletin 26, 985 (2001).

15 J. B. Kortright, et al., Phys Rev B 64, 092401 (2001).

16 J. P. Hannon, G. T. Trammell, M. Blume, and D. Gibbs, Phys. Rev. Lett. 61, 1245 (1988).

17 J. B. Kortright and S. K. Kim, Phys. Rev. B 62, 12216 (2000).

18 C. T. Chen, et al., Phys. Rev. Lett. 75, 152 (1995).

19 J. F. Ankner and G. P. Felcher, J. Magn. Magn. Mater. 200, 741 (1999).

20 R. M. Osgood III, et al., J. Magn. Magn. Mater. 198, 698 (1999).

21 M. S. Pierce, et al., Phys. Rev. Lett. 90, 175502 (2003). 
Table 1. The integrated gain (AF intensity) and loss (F intensity) in $\Delta I_{\text {lin }}$ near the $1 / 2$ and $1^{\text {st }}$ order positions, respectively, normalized by the integrated intensity of the $1^{\text {st }}$ order peak of $I_{\text {lin }}(H=0)$.

\begin{tabular}{|c|c|c|}
\hline Sample & AF intensity & F intensity \\
\hline$h c p-C o, 298 \mathrm{~K}$ & 0.006 & -0.024 \\
\hline$\varepsilon-\mathrm{Co}, 298 \mathrm{~K}$ & 0.017 & -0.009 \\
\hline$\varepsilon-\mathrm{Co}, 32 \mathrm{~K}$ & 0.020 & -0.010 \\
\hline
\end{tabular}
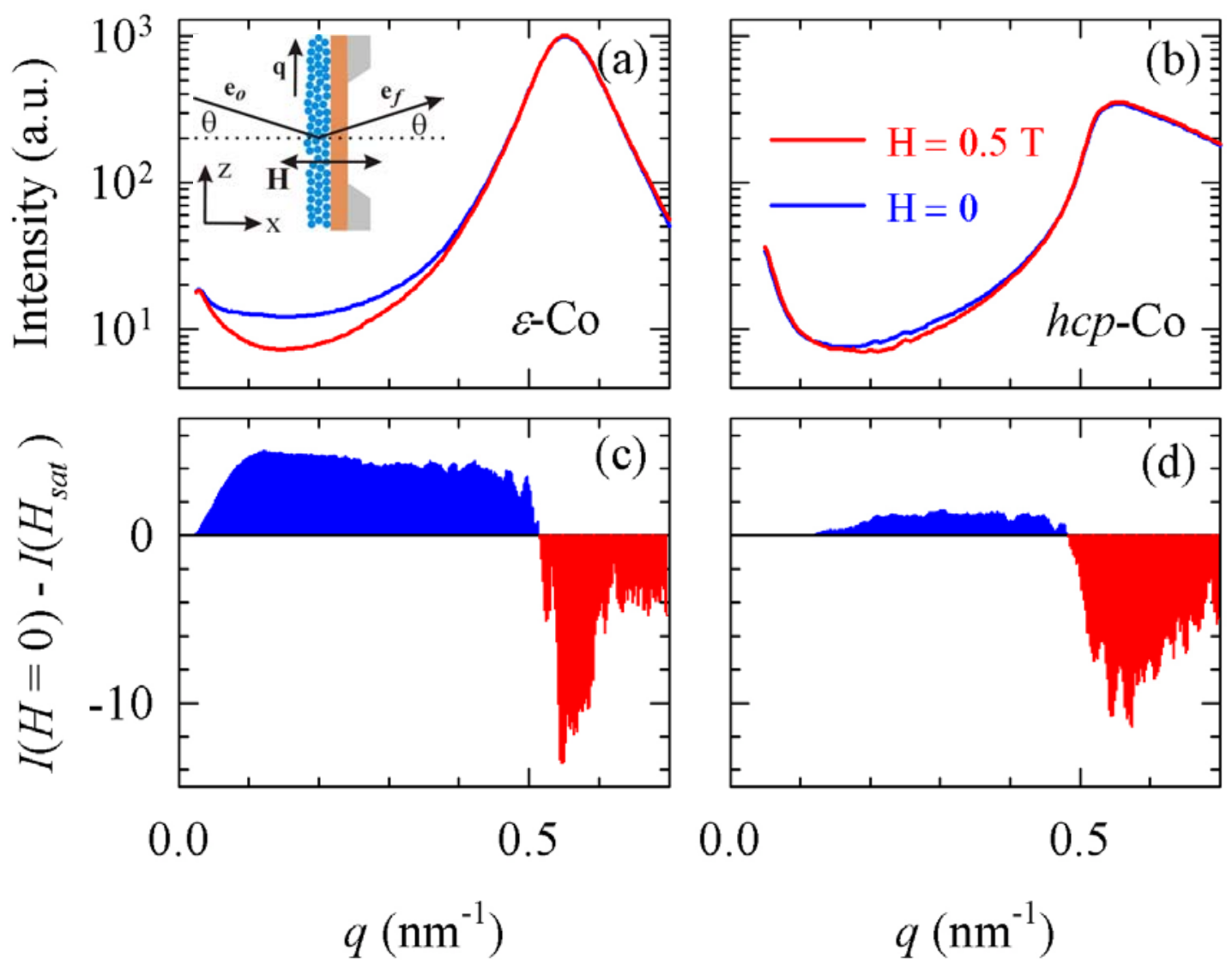

0.0

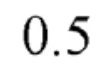

$q\left(\mathrm{~nm}^{-1}\right)$

Figure 1. (color online) (a) and (b) show $q$-resolved intensities for the $\varepsilon$ - and hcp-Co samples measured with linear polarization at saturation and remanence. (c) and (d) show the field-dependent difference for the samples above. 

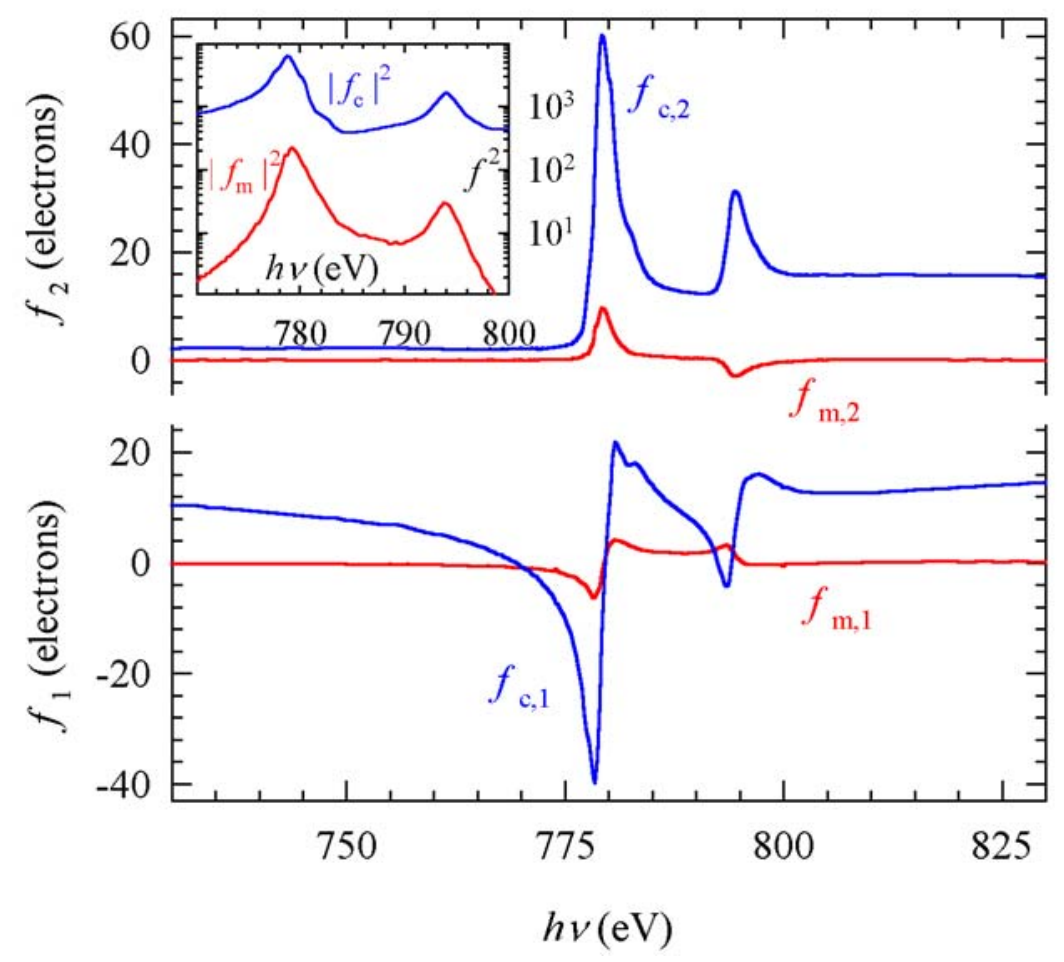

Figure 2. (color online) Real $\left(f_{1}\right)$ and imaginary $\left(f_{2}\right)$ parts of the charge and magnetic scattering factors for the $\varepsilon$-Co sample. The inset shows the relative strengths of pure charge and magnetic scattering intensities. 


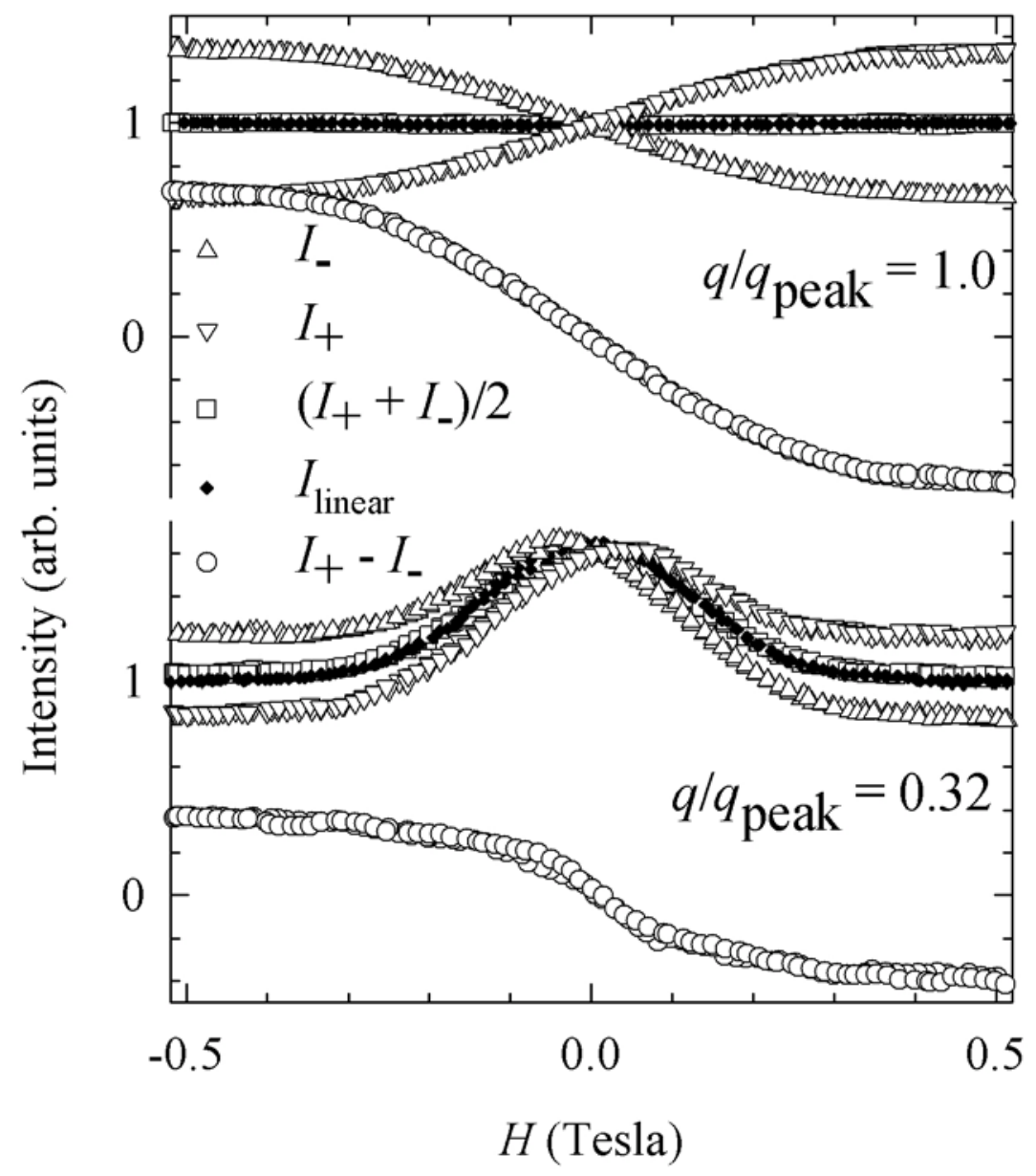

Figure 3. $298 \mathrm{~K}$ scattering loops at indicated $q$ values using linear and opposite circular polarization for the $\varepsilon$-Co sample. Also shown are the average and difference of opposite circular loops. Data are normalized so that $I_{\text {lin }}$ and $\left(I_{+}+I_{-}\right) / 2$ equal 1 at saturation. 


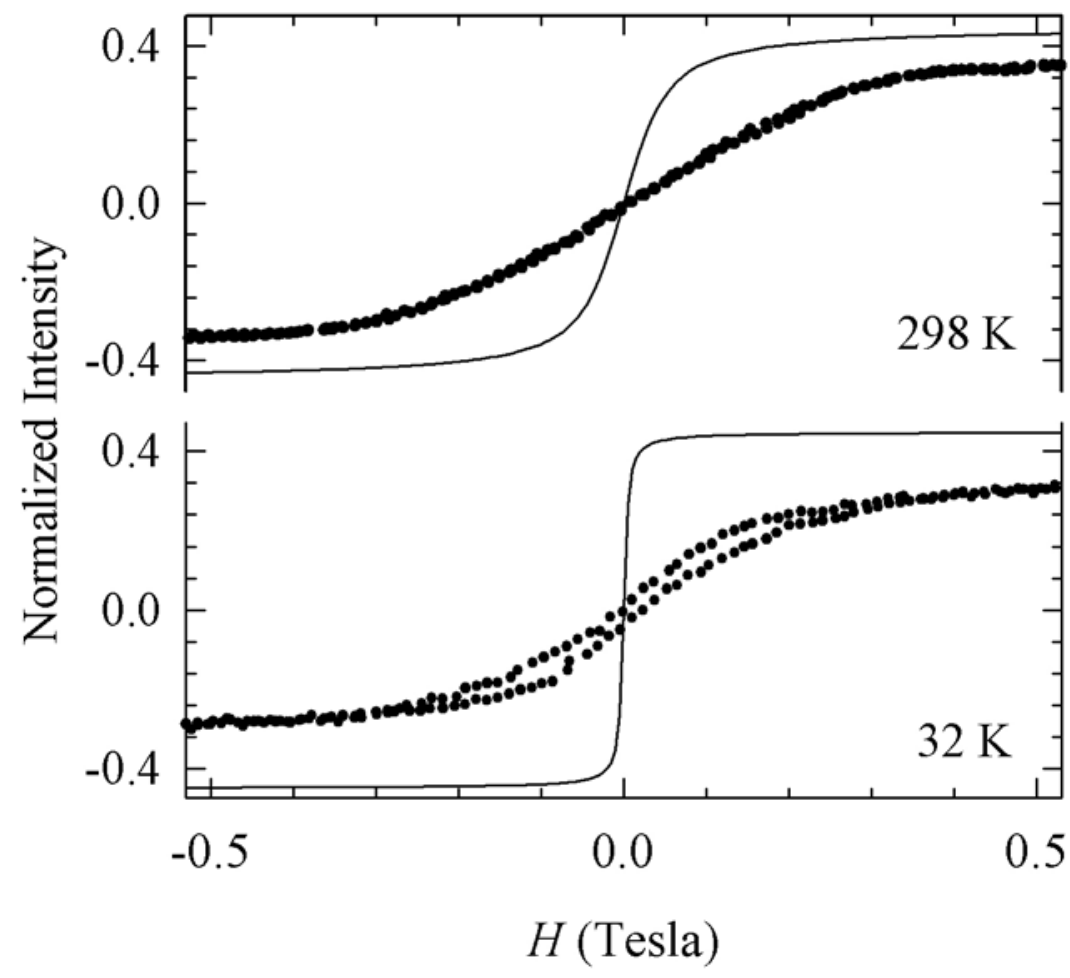

Figure 4. Scattering hysteresis loops above and below $T_{B}$ for $\varepsilon$-Co sample (symbols), and simulated loops for non-interacting, super-paramagnetic particles having $22,000 \mu_{B}$ moment (lines). 